\title{
O Estresse Térmico Visto Como Um Risco Ocupacional
}

\author{
Victor Cupertino Rosa \\ 31vrosa@gmail.com \\ Universidade Tecnológica Federal do \\ Paraná (UTFPR), Ponta Grossa \\ Paraná, Brasil. \\ Luiz Eduardo Melo Lima \\ lelima@utfpr.edu.br \\ Universidade Tecnológica Federal do \\ Paraná (UTFPR), Ponta Grossa, \\ Paraná, Brasil.
}

\begin{abstract}
RESUMO
Com a existência de riscos ocupacionais específicos, as questões de saúde e segurança do trabalhador têm sido um elemento de destaque na gestão do negócio, sendo um diferencial competitivo para as indústrias. Dentre os inúmeros riscos ocupacionais existentes, o estresse térmico é objeto deste estudo, pois tal estriç̧ão pode interferir no rendimento da produção e expor o trabalhador ao risco de acidentes, doenças ocupacionais e morte. Este trabalho tem por objetivo apresentar uma revisão sobre estresse térmico a partir dos estudos de diversos pesquisadores, bem como seus resultados, com a finalidade de fornecer uma base de conhecimento a sociedade e as organizações. Com o avanço das pesquisas, observa-se que muitos estudos têm sido realizados não só em câmaras climatizadas, mas também em situações reais do cotidiano, com pessoas desempenhando suas atividades rotineiras. Por meio de tais pesquisas, é possivel observar que o homem, sendo um animal homeotérmico, precisa manter sua temperatura constante, sofrendo influência direta da temperatura do ambiente em que se encontra. $O$ risco de graves doenças provocadas pelo calor pode ser diminuído por meio de várias medidas para combatê-las, como a aclimatação ao calor, o controle da exposição ao estresse térmico e a manutenção da hidratação. Antes de qualquer índice ou norma, defende-se que todas as organizações devem enxergar o estresse térmico como um risco ocupacional de fato, buscando as condições que mais satisfaçam o homem com relação às suas sensações térmicas, trazendo maior disposição para o trabalho e a consequente melhoria da qualidade de vida.
\end{abstract}

PALAVRAS-CHAVE: Conforto térmico. Estresse térmico. Risco ocupacional. 


\section{INTRODUÇÃO}

A Revolução Industrial trouxe inúmeras alterações na forma em que a classe trabalhadora passou a executar suas tarefas, gerando longas jornadas de trabalho, locais sem segurança, máquinas inadequadas e ambientes sem qualquer planejamento (GALLOIS, 2002).

E esse desrespeito ao ambiente no qual o trabalhador executa suas tarefas ainda não se extinguiu com o passar dos anos, nem com o advento da internet $\mathrm{e}$ da sociedade globalizada. Um exemplo disto pode ser observado nos anos de 2012 a 2013, quando treze trabalhadores morreram nas indústrias dos Estados Unidos nessa época, por consequência da sobrecarga térmica, segundo o Centro de Controle e Prevenção de Doenças (CDC, do inglês Center for Disease Control and Prevention) (OSHA, 2018).

Portanto, com a existência de riscos ocupacionais específicos, as questões de saúde e segurança do trabalhador têm sido um elemento de destaque na gestão do negócio, sendo um diferencial competitivo para as indústrias nos dias atuais.

Neste ínterim, dentre os inúmeros riscos ocupacionais existentes no ambiente de trabalho, o estresse térmico é objeto deste estudo, pois o mesmo pode interferir no rendimento da produção e expor o trabalhador ao risco de acidentes, doenças ocupacionais e morte (LINDSLEY; CADORETTE, 2015).

De acordo com Gambrell (2002), pessoas que trabalham em ambientes muito quentes encaram desafios fisiológicos que podem afetar o desenvolvimento de suas atividades e, adicionalmente, podem também ser incididos por perdas térmicas sérias e até risco de morte. A elevação da temperatura corporal a níveis críticos acarreta na incidência de doenças térmicas, em particular, a exaustão térmica e o EHS (Exertional Heat Stroke ou insolação por esforço), que são duas formas de insolação que atingem trabalhadores expostos a situações de estresse térmico.

Destaca-se a importância em se discutir o estresse térmico e, em oposição a isto, o conforto térmico do trabalhador, levando-se em conta o que ensina Lamberts (2016). Segundo ele, as unidades industriais distinguem que um aumento da satisfação ambiental favorece os índices de produtividade dos colaboradores, assim como um controle individual dos sistemas de climatização oferece um aumento de produtividade de $2,8 \%$ a $8,6 \%$, conforme o tipo de atividade. $\mathrm{O}$ conforto térmico tem sido objeto de estudo de diversas pesquisas realizadas recentemente.

Keim, Guisto e Sullivan Jr. (2002) apresentaram uma revisão de normas publicadas, limites e recomendações referentes às práticas de trabalho com relação à segurança individual e cargas térmicas suportadas pelos indivíduos. Eles abordaram neste estudo diversos fatores relacionados ao estresse térmico que afetam a saúde do trabalhador, bem como a produtividade das organizações. Constata-se, que os indivíduos estão sujeitos a mudanças fisiológicas termorreguladoras para adaptação às condições térmicas do ambiente de trabalho (VANGELOVA et al., 2008).

A partir da realização de uma auditoria de segurança e saúde do trabalho numa indústria de alimentos e bebidas, Lacerda et al. (2005) identificaram problemas resultantes do desconforto térmico. De acordo com relatos dos 
trabalhadores, foram observados cerca de oito desmaios, na área dos canhões de pipoca, em pessoas do sexo masculino de 25 a 30 anos, devido aos níveis de temperatura acima dos limites de tolerância para exposição ao calor. Problema semelhante ocorreu na área das drajadeiras, porém, com pessoas do sexo feminino da faixa etária de 40 anos. A partir desta auditoria, foi sugerida a realização de ações visando o atendimento das normas para garantia da segurança e saúde dos trabalhadores, para redução de custos com causas trabalhistas, bem como a eliminação ou minimização de situações de risco.

Santos, Fialho e Cavalcante (2011) realizaram uma análise ergonômica de operadores de duas máquinas de esmaltagem utilizando tecnologias diferentes. Eles observaram situações de desconforto térmico em dias com temperatura mais elevadas, devido à falta de janelas ou sistema de climatização do setor. 0 ambiente é muito amplo de tal forma que inviabiliza a instalação de um sistema de climatização, segundo informações da gerência.

Moura e Xavier (2012) realizaram um estudo para analisar a sensação térmica a partir de um estudo de caso em uma empresa de pequeno porte, identificando as ações dos indivíduos para garantir seu conforto térmico. A partir da aplicação de questionários, eles observaram a importância do uso correto de roupas apropriadas para o ambiente de trabalho, sendo considerado como um equipamento de proteção individual. Além disso, o estudo mostrou que a sensação térmica dos indivíduos é dependente da atividade desempenhada e da sensibilidade, como consequência do isolamento térmico de vestimenta.

Por meio da realização de uma avaliação das condições de trabalho dos agentes de bagagem e operadores de rampa de um aeroporto brasileiro, Santos e Monteiro (2017) identificaram os fatores de risco associados às atividades desenvolvidas por esses trabalhadores, de modo a propor soluções ergonômicas viáveis de serem aplicadas pela empresa. A análise do conforto térmico do ambiente de trabalho demonstrou que a temperatura média apresentou valores $11 \%$ maiores que os limites recomendados pela legislação vigente. Além disto, foi observado que o Índice de Bulbo Úmido Termômetro de Globo (IBUTG) também estava fora dos limites de tolerância estabelecidos. De modo mais especifico, o IBUTG médio estava 2,65\% e 8,29\% acima dos limites de exposição de acordo com a legislação brasileira, para os agentes de bagagem e para os operadores de rampa, respectivamente.

Albuquerque et al. (2018) realizaram uma avaliação do conforto térmico em uma indústria por meio da aplicação de questionários específicos para avaliação térmica, bem como a partir de medições das variáveis ambientais de interesse: umidade relativa, temperatura e velocidade do ar. Os indivíduos foram questionados sobre fatores que influenciam a sensação de frio no inverno e fatores que influenciam a sensação de calor no verão. Eles identificaram que as temperaturas elevadas medidas na indústria e a falta de ventilação natural estavam em condições não satisfatórias e conseguiram, a partir da realização de ações imediatas por meio da instalação de exaustores, uma redução de $2,9^{\circ} \mathrm{C}$ no IBUTG, melhorando assim as condições dos trabalhadores, resultando num aumento da produtividade.

Para que se possa compreender como o fator térmico influi em diversas áreas da produção - industrial e intelectual -, são apresentados neste trabalho estudos de diversos pesquisadores sobre o tema e seus resultados. 
Sendo assim, este estudo sobre o risco ocupacional relacionado ao estresse térmico tem por objetivo apresentar uma revisão sobre o tema, para oferecer a sociedade e as organizações uma base de conhecimento sobre o assunto. Deste modo, podem ser desenvolvidas melhorias no conforto térmico de trabalho, até como forma de motivação e garantia da própria vida dos colaboradores, proporcionando ambientes e condições de trabalho que atendam às legislações vigentes, oferecendo qualidade de vida e satisfação aos trabalhadores.

\section{REFERENCIAL TEÓRICO}

Este estudo se caracteriza como uma pesquisa bibliográfica, já que, consoante Cervo, Silva e Bervian (2016), este tipo de pesquisa revela explicitamente o universo de contribuições científicas de autores sobre um tema específico.

Portanto, a partir daqui poder-se-á ler o que os mais diversos autores escreveram sobre o tema, procurando discorrer sobre a temperatura humana, o calor e a neutralidade térmica, bem como o conforto e o estresse térmicos.

\section{TEMPERATURA HUMANA}

O homem é um animal homeotérmico, ou seja, seu organismo é conservado a uma temperatura interna constante. Esta temperatura é de $37^{\circ} \mathrm{C}$ (MORRISON; NAKAMURA, 2019), com tolerâncias muito próximas - de $36,1^{\circ} \mathrm{C}$ a $37,2^{\circ} \mathrm{C}-$, sendo $32^{\circ} \mathrm{C}$ e $42^{\circ} \mathrm{C}$ os respectivos limites inferior e superior para sobrevivência, se doente (FROTA; SCHIFFER, 2005).

Segundo Guyton e Hall (2006), o corpo humano é termicamente dividido em um núcleo central quente e uma camada externa mais fria. Nem todas as partes possuem a mesma temperatura e também não são afetadas pelos mesmos fatores. A temperatura corporal central, ou seja, a temperatura dos tecidos mais profundos do corpo permanece constante, com variação fisiológica de aproximadamente $0,6^{\circ} \mathrm{C}$, a não ser em caso de febre.

O meio ambiente influência na temperatura da camada externa, por isto esta não é regulada dentro dos mesmos limites da temperatura interna do corpo (WIDMAIER; RAFF; STRANG, 2006; KANG et al., 2018).

A manutenção da temperatura interna do organismo humano relativamente constante, em ambientes cujas condições de temperatura e suas variações são as mais variadas, se faz por intermédio de seu aparelho termorregulador, que comanda a redução dos ganhos ou o aumento das perdas de calor por meio de alguns mecanismos de controle (FOHR, 2015; MORRISON; NAKAMURA, 2019). A coordenação entre a produção e liberação do calor orgânico interno, apesar de ser o meio natural de controle de perdas de calor pelo organismo, representa um esforço extra e, por conseguinte, uma queda de potencialidade de trabalho (FROTA; SCHIFFER, 2005).

Visto que os interesses deste estudo são o conforto e o estresse térmicos, faz-se necessário uma pesquisa sobre o que é o calor, para que se entenda a importância desta forma de energia. 
Calor é a forma de energia que se transfere de um sistema para outro em virtude de uma diferença de temperatura entre os mesmos, ocorrendo por meio de várias formas: condução, convecção e radiação (CATAl, 2013). Salienta-se que a quantidade desta energia transmitida é determinada pela variação da temperatura do corpo que forneceu ou recebeu calor, bem como da natureza e da quantidade de matéria presente (FROTA; SCHIFFER, 2005).

Sobre isto, Frota e Schiffer (2005) ensinam que a avaliação do calor a que um indivíduo está exposto é importante. Assim sendo, a temperatura do corpo e as condições ambientais devem ser levantadas, pois influenciam diretamente nas trocas térmicas entre o corpo humano e o meio ambiente (WANG; HU, 2018; ZHANG; CAO; ZHU, 2019).

Portanto, entende-se que o corpo humano produz calor por meio de seus processos metabólicos, entretanto, é necessário que o calor produzido seja dissipado tão rapidamente quanto se produz (FROTA; SCHIFFER, 2005; WRIGHT et al., 2012). Isto por que em algumas atividades de trabalho, o ser humano é exposto ao calor excessivo, que certamente interfere na quantidade e qualidade de trabalho que o homem pode realizar.

Para um melhor entendimento dos assuntos relacionados ao conforto térmico, é necessário apresentar alguns conceitos e definições a respeito de neutralidade e conforto térmico.

\section{NEUTRALIDADE TÉRMICA}

Segundo Frota e Schiffer (2005), neutralidade térmica é: "a condição na qual uma pessoa não prefira nem mais calor nem mais frio no ambiente a seu redor".

Conforme Lamberts (2016), a neutralidade térmica é uma condição necessária, mas não suficiente, para que uma pessoa esteja em conforto térmico. Como o corpo humano é um sistema homeotérmico - que produz calor e interage continuamente com 0 ambiente para alcançar o balanço térmico (GUYTON; HALL, 2006) -, existe uma constante troca de calor entre o corpo e o meio que é regida pelas leis da física e influenciada pelos mecanismos de adaptação fisiológica, condições ambientais e fatores individuais (DEGORRE et al., 2015). Esta troca é denominada de termorregulação, que ocorre em grande parte devido à evaporação do suor (FOHR, 2015; HAILES et al., 2016).

Percebe-se então, que a sensação de conforto térmico está diretamente relacionada ao esforço realizado pelo organismo para manter o balanço térmico.

\section{CONFORTO TÉRMICO}

Lamberts (2016) ensina que os estudos de conforto térmico visam analisar e estabelecer as condições necessárias para a avaliação e concepção de um ambiente térmico adequado às atividades e ocupação humanas, bem como estabelecer métodos e princípios para uma detalhada análise térmica de um ambiente. 
Segundo a ANSI/ASHRAE Standard 55-1992, o conforto térmico é assim definido: "conforto térmico é a condição da mente que expressa satisfação com o ambiente térmico" (ASHRAE, 1992).

Gambell (2002) afirma que o conforto térmico é uma sensação complexa que sofre influência de fatores de ordem física, fisiológica e psicológica, e que, do ponto de vista puramente térmico, as condições ambientais confortáveis são aquelas que permitem ao ser humano manter constante a temperatura do corpo sem acionar, de forma perceptível, seus mecanismos termorreguladores.

Mais recentemente, foi desenvolvido um índice de avaliação do conforto térmico mais moderno, denominado PET (Physiological Equivalent Temperature Temperatura Fisiológica Equivalente). Este índice é expresso em uma escala termométrica já conhecida, no caso graus Celsius $\left({ }^{\circ} \mathrm{C}\right)$, e tem por base de cálculo a fisiologia humana (LYRA, 2007; KRUGER et al., 2018).

Assim, a condição para que exista o conforto térmico é a existência da neutralidade térmica, como já mencionado anteriormente, onde a quantidade de calor recebido deve ser igual à quantidade de calor cedido para o ambiente.

\section{ESTRESSE TÉRMICO}

A palavra estresse é derivada da palavra em inglês stress, cuja definição segundo o dicionário Michaelis (2018) é: "um estado de tensão mental ou emocional ou a tensão resultante de circunstâncias adversas ou muito exigentes".

Consoante Lamberts (2016, p. 69), stress também pode ser conceituado como: ação inespecífica dos agentes e influências nocivas (frio ou calor excessivos, infecção, intoxicação e emoções violentas tais como: inveja, ódio, medo, etc.), que causam reações típicas do organismo, tais como síndrome de alerta e síndrome de adaptação.

Assim, o estresse térmico é o estado onde tanto o sistema fisiológico quanto o sistema psicológico são atingidos pela temperatura do ambiente em que se localiza, quando esta temperatura encontra-se em níveis extremos e muito exigentes. $O$ estresse térmico pode ser considerado ainda como resultado da diminuição da capacidade do ser humano de manter sua homeotermia (JOSIPOVIC; LUDWIG, 2012).

Vários teóricos demonstram como o estresse térmico é estudado tanto para fins científicos - o pesquisador possui papel de descobrir intensidade, natureza e origem do estresse térmico de um dado ambiente, normalmente industrial, com trabalhadores sujeitos às possíveis situações de estresse térmico a serem analisadas; quanto para fins práticos - onde os gestores são responsáveis por reduzir os riscos aos quais os trabalhadores podem estar sujeitos no ambiente de trabalho (LAMBERTS, 2016).

No Brasil, existem normas regulamentadoras exigindo que os ambientes sejam avaliados e adequados termicamente ao homem, seguindo também o princípio básico da Ergonomia. É o caso da Norma Regulamentadora 17 Ergonomia, onde se afirma que "as condições ambientais de trabalho devem estar adequadas às características psicofisiológicas dos trabalhadores e à natureza do trabalho a ser executado" (MINISTÉRIO DO TRABALHO, 2007). 
$O$ estresse térmico pode acontecer em ambientes frios ou quentes (LAMBERTS, 2016), sendo que as avaliações de estresse térmico possuem dois objetivos primários, que são determinar a natureza, e assim, as causas do estresse térmico em um dado ambiente, e mensurar e avaliar a intensidade do estresse térmico, prevendo, deste modo, as suas consequências para a saúde dos trabalhadores.

Epstein e Moran (2006) apresentaram uma revisão sobre diversos índices para avaliação de estresse térmico, classificando-os em três grupos: racionais empíricos e diretos. Eles recomendaram o uso do DI (Discomfort Index - Índice de Desconforto) como um indicador universal de estresse térmico. De Freitas e Grigorieva (2015) documentaram e classificaram rigorosamente todos os índices de conforto térmico encontrados na literatura a fim de facilitar a localização e comparação. Lamberts (2016) listou cronologicamente os principais índices para avaliação de estresse térmico, conforme apresentado no Quadro 1.

Quadro 1 - Lista cronológica de índices para avaliação de estresse térmico

\begin{tabular}{|c|c|c|c|}
\hline Índice & Descrição & Autor & Ano \\
\hline ET & $\begin{array}{l}\text { Effective Temperature Scales - Escalas } \\
\text { de Temperatura Efetiva }\end{array}$ & Houghten e Yaglou & 1923 \\
\hline W & Skin Wettedness - Umidade da Pele & Gagge & 1937 \\
\hline TAR & $\begin{array}{l}\text { Thermal Acceptance Ratio - Relação de } \\
\text { Aceitação Térmica }\end{array}$ & Plummer & 1945 \\
\hline CET & $\begin{array}{l}\text { Corrected Effective Temperature - } \\
\text { Temperatura Efetiva Corrigida, } \\
\text { adaptado do índice ET }\end{array}$ & Bedford & 1946 \\
\hline P4SR & $\begin{array}{l}\text { Predicted Four Hour Sweat Rate - Taxa } \\
\text { de Suor Estimada para } 4 \text { Horas }\end{array}$ & McArdle & 1947 \\
\hline $\mathrm{HSI}$ & $\begin{array}{l}\text { Heat Stress Index - Índice de Estresse } \\
\text { Térmico }\end{array}$ & Belding e Hatch & 1955 \\
\hline WBGT & $\begin{array}{l}\text { Wet Bulb Globe Temperature - Índice de } \\
\text { Bulbo Úmido Termômetro de Globo }\end{array}$ & Yaglou e Minard & 1957 \\
\hline TSI & $\begin{array}{l}\text { Thermal Stress Index - Índice de Tensão } \\
\text { Térmica }\end{array}$ & Lee & 1958 \\
\hline \multirow{2}{*}{ DI } & \multirow{2}{*}{$\begin{array}{l}\text { Discomfort Index - Índice de } \\
\text { Desconforto }\end{array}$} & Thom & 1959 \\
\hline & & Adaptado por Sohar & 1962 \\
\hline RSI & $\begin{array}{l}\text { Relative Stress Index - Índice Relativo de } \\
\text { Tensão }\end{array}$ & Lee e Henschel & 1963 \\
\hline $\begin{array}{l}\text { ITS ou } \\
\text { SW req }\end{array}$ & $\begin{array}{l}\text { Index of Thermal Stress or Required } \\
\text { Sweat Rate - Índice de Estresse Térmico } \\
\text { ou Taxa Requerida de Suor }\end{array}$ & Givoni & 1963 \\
\hline $\mathrm{ET}^{*}$ & $\begin{array}{l}\text { New Effective Temperature - Nova } \\
\text { Temperatura Efetiva }\end{array}$ & Gagge & 1971 \\
\hline PSI & $\begin{array}{l}\text { Physiological Strain Index - Índice de } \\
\text { Tensão Fisiológica }\end{array}$ & Moran, Shitzer e Pandolf & 1998 \\
\hline MDI & $\begin{array}{l}\text { Modified Discomfort Index - Índice } \\
\text { Modificado de Desconforto }\end{array}$ & Moran e Pandolf & 1999 \\
\hline ESI & $\begin{array}{l}\text { Environmental Stress Index - Índice de } \\
\text { Stress Ambiental }\end{array}$ & $\begin{array}{l}\text { Moran, Pandolf, Shapiro, } \\
\text { Heled, Shani, Mathew e } \\
\text { Gonzalez }\end{array}$ & 2001 \\
\hline
\end{tabular}

Fonte: Autoria própria (2019). 
No Brasil, o WBGT (Wet Bulb Globe Temperature) é conhecido como IBUTG (Índice de Bulbo Úmido Termômetro de Globo), apresentado na Norma Regulamentadora 15 - Atividades e Operações Insalubres, em seu Anexo 3 Limites de Tolerância para Exposição ao Calor (MINISTÉRIO DO TRABALHO, 2014).

Depois de conhecer o objeto deste estudo, torna-se importante neste momento, observar o que outros pesquisadores descobriram em campo.

\section{METODOLOGIA}

No presente trabalho, o levantamento de estudos da literatura relacionados ao tema foi realizado por meio da pesquisa de artigos científicos, utilizando combinações de palavras-chaves em diversas bases de dados conhecidas no portal "Periódicos da Capes". As combinações de palavras-chaves utilizadas na pesquisa, bem como as bases de dados e a quantidade de artigos encontrados são apresentadas na Tabela 1.

Tabela 1 - Quantidade de artigos científicos encontrados em bases de dados em função da combinação de palavras-chaves utilizadas na pesquisa

\begin{tabular}{ccc}
$\begin{array}{c}\text { Combinação de } \\
\text { Palavras-Chaves }\end{array}$ & Base de Dados & $\begin{array}{c}\text { Quantidade de } \\
\text { Artigos }\end{array}$ \\
"Thermal comfort", & MEDLINE/PubMed (NLM) & 130 \\
"thermal stress" and & OneFile (GALE) & 91 \\
"occupational" & Materials Science \& Engineering Database & 72 \\
& ScienceDirect Journals (Elsevier) & 70 \\
"Heat stress" and & SpringerLink & 49 \\
"occupational risk" & MEDLINE/PubMed (NLM) & 56 \\
& OneFile (GALE) & 25 \\
& Materials Science \& Engineering Database & 30 \\
& PMC (PubMed Central) & 16 \\
\hline
\end{tabular}

Fonte: Autoria própria (2019).

Após ter sido realizada a pesquisa dos artigos e a eliminação de resultados repetidos, por meio da leitura do título e do resumo de cada artigo encontrado, foram selecionados somente aqueles artigos que apresentavam estudos cuja temática estava alinhada com os objetivos do presente estudo.

As demais referências utilizadas (livros, teses, normas, etc.) foram obtidas por meio de pesquisa no portal "Google Acadêmico", usando as combinações de palavras-chaves, tanto em língua inglesa quanto em língua portuguesa.

\section{ANÁLISE DE DADOS E DISCUSSÃO DOS RESULTADOS}

De acordo com Lamberts (2016), algumas indústrias nas quais casos de estresse térmico são propícios de estarem presentes são as de metais primários (sendo alumínio, aço e ferro alguns dos principais), processamento de alimentos, energia elétrica e construção. Além destes, outros processos citados por Frota e Schiffer (2005), como vulcanização de borracha, fundição de metais, produtos em cerâmica, lavanderias, cozinhas industriais e alguns trabalhos mais pesados a céu aberto podem ser incluídos, pois além da carga térmica exercida pelo sol, o 
próprio corpo humano gera mais calor devido à atividade excessiva. Sendo assim, acredita-se que os trabalhadores que atuam no corte de cana praticam uma atividade que envolve um grande risco à saúde, pois o trabalhador está sob a exposição direta do sol, exercendo uma atividade pesada e exigindo o uso de vários equipamentos de proteção, o que aumenta o isolamento térmico da roupa do trabalhador, dificultando, portanto, a perda de calor e podendo levar à morte.

O trabalho de Barbiero (2004) foi um estudo de caso junto a trabalhadores de uma unidade de produção de uma indústria metalúrgica, para a avaliação das condições e percepções do ambiente térmico, por meio do levantamento das variáveis de influência (temperatura, velocidade e umidade do ar, temperatura média radiante, atividade metabólica e isolamento térmico da vestimenta) e parâmetros subjetivos de conforto, no período de verão, com base nas normas internacionais da série ISO (International Organization for Standardization Organização Internacional de Normalização) e as normas brasileiras do tipo NR (Norma Regulamentadora) e NBR (Norma Brasileira). Nesse estudo, a pesquisadora relatou ter comprovado que os fatores ambientais e pessoais podem influenciar nas percepções do ambiente de trabalho.

Além disto, o estudo de Barbiero (2004) demonstra que, com base em índices calculados e levantados no local e em parâmetros subjetivos relativos ao conforto térmico, mesmo não tendo sido constatado estresse térmico, o ambiente apresentou índices térmicos em desconformidade aos recomendados para conforto, sendo que as percepções levantadas pelos trabalhadores, por meio da aplicação de questionário subjetivo, confirmaram essa realidade. Ela ainda relatou que, mesmo tendo sido evidenciadas as condições de desconforto térmico, $60 \%$ dos trabalhadores aceitaram as condições térmicas do ambiente de trabalho. Além disto, o estudo informa que, mesmo aceitando o ambiente térmico, o trabalhador o percebeu "desconfortável" e "quente". Ela conclui, afirmando que se tornou evidente a inexistência de uma legislação que comporte, de fato, a realidade do ambiente térmico do trabalhador da indústria, considerando as diversidades regionais, para servir de ponto de referência a toda e qualquer pesquisa nesta área do conhecimento.

O estudo de Farias et al. (2009) teve como objetivo avaliar as condições de conforto e estresse térmicos no ambiente de trabalho de professores. Para isso, as salas de aula foram monitoradas para caracterizar as condições físicas oferecidas no cotidiano diário da escola pesquisada por eles. Além disso, apresentaram a informação de que foram registradas, dos dias 2 a 6 de junho de 2008, dados sobre temperatura do ar, temperatura de bulbo úmido, temperatura de globo, velocidade do ar e Índice de Bulbo Úmido e Termômetro de Globo (IBUTG). Posteriormente, foram calculados os índices de avaliação de conforto térmico e os valores foram comparados aos limites e faixas de conforto das normas nacionais e internacionais. Os resultados apresentaram uma situação crítica de extremo desconforto em sala de aula. O IBUTG apresentou resultado máximo de $26,4^{\circ} \mathrm{C}$. Os autores concluíram que o ambiente das salas de aula da escola estudada apresentou condições de desconforto, com provável diagnóstico de ambiente insalubre, em períodos anuais mais quentes.

Miller e Bates (2007) avaliaram os limites térmicos de trabalho (TWL Thermal Work Limit) em ambientes industriais controlados por meio do monitoramento dos sinais fisiológicos caracterizados usando tanto o IBUTG 
quanto o TWL. Eles verificaram que o TWL é mais apropriado do que o IBUTG para avaliação do estresse térmico, conforme observado em estudos anteriores.

Gosling e Araújo (2008) mediram o nível de ruído e de carga térmica as quais um operador de duas diferentes marcas de tratores esteja submetido, bem como sugeriram formas de melhorar o bem estar do operador com relação ao conforto térmico no trabalho. Eles observaram que a carga térmica produzida pelo maquinário é um fator determinante na ocorrência de doenças, como desidratação, insolação, entre outras.

Kovats e Hajat (2008) apresentaram uma revisão crítica sobre estresse térmico e saúde pública. Eles relacionaram o aumento do risco de mortalidade devido ao calor, ao envelhecimento natural de cada indivíduo, mas também a fatores associados com a vulnerabilidade social particular de cada um. Eles sugerem que as medidas de saúde pública devem promover mecanismos de aviso da ocorrência de ondas de calor, bem como promover a melhoria no conforto térmico das habitações, entre outras medidas.

Oliveira (2009), em seu estudo de caso da atividade de manutenção de linhas energizadas, queria propor uma complementação, com caráter proativo, ao método e aos procedimentos de avaliação da exposição ocupacional ao calor previstos na Norma Regulamentadora - NR 15 (MINISTÉRIO DO TRABALHO, 2014) e na Norma de Higiene Ocupacional - NHO 06 (FUNDACENTRO, 2017), respectivamente. A população escolhida nesse estudo de caso foi composta pelos eletricistas da turma de manutenção de linhas energizadas ao potencial, na tensão de 69 kV, da Companhia Energética de Alagoas (CEAL). Uma das principais constatações obtidas com as avaliações que integram esta pesquisa citada diz respeito à significativa perda hídrica por hora de trabalho ao qual pode ser submetido o organismo do trabalhador, quando a realização das tarefas exige a utilização de vestimentas especiais de proteção. Em seu estudo, o pesquisador explica que quando esta perda hídrica suplanta os limites especificados na Norma ISO 7933:1989 (ISO, 1989), conclui-se que poderão ocorrer consequências fisiológicas danosas à saúde do trabalhador. Ele destaca que, sobre esta abordagem específica, os documentos legais brasileiros são omissos.

Outro trabalho escolhido para ser apresentado aqui, como um caso prático já estudado, é o de Juliana Basso da Fonseca que, no ano de 2014, apresentou uma análise dos níveis de calor nos postos de trabalho de uma lavanderia industrial. Em sua pesquisa, Fonseca (2014) objetivou medir os níveis de temperatura nos postos de trabalho selecionados qualitativamente em uma lavanderia industrial localizada na região metropolitana de Curitiba. Segundo a pesquisadora, muitos trabalhadores passam parte de sua jornada diária expostos a condições adversas de calor que representam certos perigos para a sua segurança e saúde. A antecipação, o reconhecimento, a avaliação e o controle destes riscos devem estar presentes no Programa de Prevenção de Riscos Ambientais (PPRA) e no Programa de Controle Médico de Saúde Ocupacional (PCMSO), documentos exigidos legalmente e fundamentados nas Normas Regulamentadoras 9 e 7 do Ministério do Trabalho (MINISTÉRIO DO TRABALHO, 2017, 2013), respectivamente. Com o auxílio de um termômetro de globo, foram realizadas as medições das temperaturas e calculados os Índices de Bulbo Úmido Termômetro de Globo. Os dados obtidos foram comparados com os Limites de Tolerância para exposição ao calor, em regime de trabalho intermitente com períodos de descanso no próprio local de prestação de serviço, considerando a atividade 
como moderada, seguindo as instruções da Norma Regulamentadora 15, do Ministério do Trabalho (MINISTÉRIO DO TRABALHO, 2014). Os resultados obtidos indicaram esse posto de trabalho como insalubre. Por outro lado, outros pontos de medição apontaram a necessidade de períodos de descanso. A partir destes resultados, foram sugeridas melhorias e adequações para tornar o local de trabalho ergonômico e termicamente adequado aos colaboradores, reduzindo assim as possibilidades de acidentes de trabalho.

Cruz (2018) buscou identificar os efeitos resultantes da exposição simultânea a fatores de risco físico sobre a saúde de operadores de equipamentos na indústria de construção civil, relativos aos aspectos fisiológicos e cognitivos, a fim de desenvolver uma metodologia para análise combinada dos fatores de risco físico. Para isto, foram realizadas 50 medições ocupacionais de ruído, estresse térmico e vibrações de corpo inteiro em operadores de equipamentos da construção civil durante toda a jornada de trabalho. Também foram coletados dados de frequência cardíaca e de desempenho em teste de atenção durante a jornada de trabalho. Ele constatou que os níveis de ruído apresentaram valores abaixo dos limites estabelecidos na legislação vigente. Por outro lado, os níveis de estresse térmico e vibrações de corpo inteiro apresentaram valores acima dos limites recomendados.

Terra et al. (2019) realizaram um estudo de caso numa empresa de pequeno porte do setor alimentício, com a finalidade de identificar melhorias nas condições de conforto térmico dos trabalhadores por meio da aplicações dos conceitos de conforto térmico. Eles levantaram dados de campo usando um medidor de estresse térmico. A partir dos resultados obtidos, eles verificaram que a sensação térmica levemente fria do ambiente produzia uma perda de eficiência na capacidade produtiva dos colaboradores, podendo ocorrer riscos ocupacionais. Diante destes resultados, foram propostas algumas medidas para melhorar a sensação de conforto térmico dos colaboradores.

\section{CONSIDERAÇÕES FINAIS}

A realização deste estudo sobre o conforto e o estresse térmicos, teve por objetivo apresentar uma revisão sobre o tema, para oferecer a sociedade e as organizações uma base de conhecimento sobre o assunto. Por isto, torna-se importante destacar que o estresse térmico é um problema comum em várias indústrias e atividades laborais, pois os trabalhadores frequentemente estão expostos a temperaturas acima dos limites convencionais ou recomendados.

Assim, o estresse térmico, como um fator ambiental, pode influenciar significativamente na produtividade do trabalho executado por um operador, levando à redução do entusiasmo do trabalhador e aumentando a taxa de incidentes, que podem se tornar acidentes de trabalho.

Com o avanço das pesquisas, observou-se que muitos estudos foram realizados não só em câmaras climatizadas, mas também em situações reais do cotidiano, com pessoas desempenhando suas atividades rotineiras. Nestas pesquisas de campo, o pesquisador não interfere diretamente nas variáveis ambientais e pessoais, e as pessoas expressam suas sensações e preferências térmicas de acordo com escalas apropriadas. 
Por meio de tais pesquisas foi possível observar que o homem, sendo um animal homeotérmico, precisa manter sua temperatura constante, sofrendo influência direta da temperatura do ambiente em que se encontra. $O$ risco de graves doenças provocadas pelo calor pode ser diminuído por meio de várias medidas para combatê-las, como a aclimatação ao calor, controle da exposição ao estresse térmico e a manutenção da hidratação.

Antes de qualquer índice ou norma, defende-se que todas as organizações devem enxergar o estresse térmico como um risco ocupacional de fato, buscando as condições que mais satisfaçam o homem com relação às suas sensações térmicas, trazendo maior disposição para o trabalho e a consequente melhoria da qualidade de vida.

Em relação a estudos anteriores, o presente trabalho apresenta alguns resultados de pesquisas mais recentes, que levantam novos questionamentos e propõem novas soluções para o problema estudado, considerando diversos aspectos. Este tipo de trabalho apresentado aqui, apesar das suas limitações, tem uma grande importância para que se mantenha o interesse e o foco das pesquisas no tema estudado, para que desta forma, novos estudos possam ser realizados contribuindo com o desenvolvimento da sociedade como um todo. 


\title{
Thermal stress as an occupational risk
}

\begin{abstract}
With the existence of specific occupational hazards, worker health and safety issues become a prominent element in business management, being a competitive differential for the industries. Among the many occupational hazards that exist, thermal stress was the object of this study, since such strictness can interfere with the production yield and expose the worker to the risk of accidents, occupational diseases, and death. This work aims to present a review on thermal stress from the studies of several researchers, as well as their results, with the purpose of providing a knowledge base to society and organizations. It was observed that, with the research advancement, many studies were carried out not only in acclimatized chambers but also in real situations of daily life, with people performing their routine activities. Through such research, it was possible to observe that human, being a homoeothermic animal, must maintain its constant temperature, suffering direct influence of the environment temperature in which it is. The serious heat risk illness can be reduced by various measures to combat them, such as acclimatization to heat, exposure control to thermal stress and hydration maintenance. Before any index or norm, it is argued that all organizations should view thermal stress as a real occupational hazard, seeking the conditions that satisfy the man with respect to their thermal sensations, bringing greater disposition to work and consequent life quality.
\end{abstract}

KEYWORDS: Thermal comfort. Thermal stress. Occupational risk. 


\section{REFERÊNCIAS}

ALBUQUERQUE, D. F. F.; DA NÓBREGA, J. A.; DE MELO, R. H. F.; PIRES, C. A. Gerenciamento de riscos físicos em ambiente fabril de calçados. R. Gest. Industr., Ponta Grossa, v. 14, n. 1, p. 19-35, jan./mar. 2018. DOI: 10.3895/gi.v14n1.5749. Disponível em: <http://periodicos.utfpr.edu.br/revistagi/article/view/5749>.

Acesso em: 20 maio 2018.

ASHRAE. ANSI/ASHRAE Standard 55-1992: Thermal Environmental Conditions for Human Occupancy. Atlanta, GA, USA: American Society of Heating, Refrigerating and Air-Conditioning Engineers - ASHRAE, 1992.

BARBIERO, Miriam. Avaliação das percepções quanto ao ambiente térmico em uma indústria metalúrgica: um estudo de caso. 2004. 139 f. Dissertação (Mestrado Profissionalizante em Engenharia) - Escola de Engenharia, Universidade Federal do Rio Grande do Sul, Porto Alegre. 2004. Disponível em: <http://hdl.handle.net/10183/10161>. Acesso em: 14 maio 2018.

CATAI, I. Ergonomia: Projeto e Produção. São Paulo: Edgard Blücher, 2013.

CERVO, A. L.; SILVA, R.; BERVIAN, P. A. Metodologia Científica. 6. ed. São Paulo: Atlas, 2016.

CRUZ, Felipe Mendes da. Avaliação dos efeitos sinérgicos sobre os trabalhadores expostos a fatores de risco físico em simultâneo. 2018. 148 f. Tese (Doutorado em Engenharia Industrial e de Sistemas) - Escola de Engenharia, Universidade do Minho, Braga, Portugal. 2018. Disponível em: <http://hdl.handle.net/1822/58970>. Acesso em: 30 mar. 2019.

DE FREITAS, C. R.; GRIGORIEVA, E. A. A comprehensive catalogue and classification of human thermal climate indices. International Journal of Biometeorology, Springer, v. 59, n. 1, p. 109-120, 2015. DOI: 10.1007/s00484014-0819-3. Disponível em: <http://link.springer.com/article/10.1007/s00484014-0819-3>. Acesso em: 18 abr. 2019.

DEGORRE, C. et al. A mean body temperature of $37^{\circ} \mathrm{C}$ for incubated preterm infants is associated with lower energy costs in the first 11 days of life. Acta Paediatrica, Wiley, v. 104, n. 6, p. 581-588, 2015. DOI: 10.1111/apa.12965. Disponível em: <http://onlinelibrary.wiley.com/doi/full/10.1111/apa.12965>. Acesso em: 20 mar. 2019. 
EPSTEIN, Y.; MORAN, D. S. Thermal comfort and the heat stress indices. Industrial Health, Kawasaki, Kanagawa, Japan, v. 44, n. 3, p. 388-398, 2006. DOI:

10.2486/indhealth.44.388. Disponível em:

<http://www.jstage.jst.go.jp/article/indhealth/44/3/44_3_388/_article>. Acesso

em: 20 mar. 2019.

FARIAS, Patrícia Marins; NERY, Jussana Maria Fahel Guimarães; FREIRE, Márcia Rebouças; MORAES, Luiz Roberto Santos. Conforto térmico e ambiente de trabalho docente em uma escola municipal de Salvador-BA. In: X ENCONTRO NACIONAL E VI ENCONTRO LATINO AMERICANO DE CONFORTO NO AMBIENTE CONSTRUÍDO, Natal, 16 a 18 set. 2009. Anais... Natal; Universidade Federal do Rio Grande do Norte - UFRN, 2009.

FOHR, J. P. Heat and Moisture Transfer between Human Body and Environment. Hoboken, NJ, USA: John Wiley \& Sons, Inc., 2015. crossref

FONSECA, Juliana Basso da. Análise dos níveis de calor nos postos de trabalho de uma lavanderia industrial. 2014. $34 \mathrm{f}$. Trabalho de Conclusão de Curso (Especialização em Engenharia de Segurança do Trabalho) - Departamento Acadêmico de Construção Civil, Universidade Tecnológica Federal do Paraná, Curitiba, 2014. Disponível em:

<http://repositorio.roca.utfpr.edu.br/jspui/handle/1/3532>. Acesso em: 13 maio 2018.

FROTA, A. B.; SCHIFFER, S. R. Manual de Conforto Térmico. 8. ed. São Paulo: Studio Nobel, 2005.

FUNDACENTRO. Norma de Higiene Ocupacional - NHO 06: Avaliação da Exposição Ocupacional ao Calor. 2. ed. GIAMPAOLI, Eduardo; SAAD, Irene Ferreira de Souza Duarte; DA CUNHA, Irlon de Ângelo; SHIBUYA; Elisa Kayo (Orgs.). São Paulo: Fundacentro, Ministério do Trabalho, 2017. Disponível em: $<$ http://www.fundacentro.gov.br/biblioteca/normas-de-higieneocupacional/publicacao/detalhe/2018/1/nho-06-avaliacao-da-exposicaoocuacional-ao-calor>. Acesso em: 10 maio 2018.

GALLOIS, Nelson Simões Pires. Análise das condições de stress e conforto térmico sob baixas temperaturas em indústrias frigoríficas de Santa Catarina. 2002. 125 f. Dissertação (Mestrado em Engenharia de Produção) - Programa de Pós-Graduação em Engenharia de Produção, Universidade Federal de Santa Catarina, Florianópolis, 2002. Disponível em: <http://www.labeee.ufsc.br/node/207>. Acesso em: 14 maio 2018. 
GAMBRELL, R. C. Doenças térmicas e exercício. In: LILLEGARD, W. A.; BUTCHER, J. D.; RUCKER, K. S. (Orgs.). Manual de Medicina Desportiva: Uma Abordagem Orientada aos Sistemas. São Paulo: Manole, p. 457-464, 2002.

GOSLING, M.; ARAÚJO, G. C. D. Saúde física do trabalhador rural submetido a ruídos e à carga térmica: um estudo em operadores de tratores. Mundo da Saúde, São Paulo, v. 32, n. 3, p. 275-86, 2008. DOI: 10.15343/01047809.2008323275286. Disponível em: <http://www.revistamundodasaude.com.br/assets/artigos/2008/63/275286. pdf>. Acesso em: 30 mar. 2019.

GUYTON, A. C.; HALL, J. E. Tratado de Fisiologia Médica. 11. ed. Rio de Janeiro: Guanabara Koogan, 2006.

HAILES, W. S.; CUDDY, J. S.; COCHRANE, K.; RUBY, B. C. Thermoregulation during extended exercise in the heat: comparisons of fluid volume and temperature. Wilderness \& Environmental Medicine, Elsevier, v. 27, n. 3, p. 386-392, 2016. DOI: 10.1016/j.wem.2016.06.004. Disponível em: <http://www.sciencedirect.com/science/article/pii/S1080603216301053>. Acesso em: 18 abr. 2019.

ISO. ISO 7933:1989: Hot Environments - Analytical Determination and Interpretation of Thermal Stress Using Calculation of Required Sweat Rate. International Organization for Standardization - ISO. 1989. Disponível em: <http://www.iso.org/standard/14903.html>. Acesso em: 10 maio 2018.

JOSIPOVIC, S.; LUDWIG, E. Heat Stress: Causes, Treatment and Prevention. Hauppauge, NY, USA: Nova Science Publishers, Inc., 2012.

KANG, Z.; UDAYRAJ; WAN, X.; WANG, F. A new hybrid personal cooling system (HPCS) incorporating insulation pads for thermal comfort management: Experimental validation and parametric study. Building and Environment, Elsevier, v. 145, p. 276-289, 2018. DOI: 10.1016/j.buildenv.2018.09.033. Disponível em: <http://www.sciencedirect.com/science/article/pii/S0360132318305912>. Acesso em: 18 abr. 2019. crossref

KEIM, S.; GUISTO, J.; SULLIVAN JR., J. Environmental thermal stress. Annals of Agricultural and Environmental Medicine, Lublin, Poland, v. 9, n. 1, p. 1-15, 2002. Disponível em: <http://www.aaem.pl/Environmental-thermal-stress,72764,0,2.html>. Acesso em: 30 mar. 2019. 
KOVATS, R. S.; HAJAT, S. Heat stress and public health: a critical review. Annual Review of Public Health, Palo Alto, CA, USA, v. 29, n. 1, p. 41-55, 2008. DOI: 10.1146/annurev.publhealth.29.020907.090843. Disponível em: <http://www.annualreviews.org/doi/10.1146/annurev.publhealth.29.020907.090 843>. Acesso em: 30 mar. 2019.

KRUGER, E. L.; ROSSI, F. A.; CRISTELI, P. S.; SOUZA, H. A. Calibração do índice de conforto para espaços externos Physiological Equivalent Temperature (PET) para Curitiba. Ambiente Construído, Porto Alegre, vol. 18, n. 3, pp. 135-148, 2018. DOI: 10.1590/s1678-86212018000300272. Disponível em: $<$ http://www.scielo.br/scielo.php?script=sci_arttext\&pid=S167886212018000300135\&lng=en\&nrm=iso>. Acesso em: 18 abr. 2019.

LACERDA, C. A.; CHAGAS, C. E. P.; BARBOSA, C. C.; CABRERA, J. V. D.; DE FARIAS, J. V. Auditoria de segurança e saúde do trabalho em uma indústria de alimentos $\mathrm{e}$ bebidas. R. Gest. Industr., Ponta Grossa, v. 1, n. 2, p. 43-57, 2005. DOI: 10.3895/S1808-04482005000200004. Disponível em: <http://periodicos.utfpr.edu.br/revistagi/article/view/164>. Acesso em: 20 maio 2018.

LAMBERTS, R. Conforto e Stress Térmico. Apostila. Florianópolis: Laboratório de Eficiência Energética em Edificações, Departamento de Engenharia Civil, Centro Tecnológico, Universidade Federal de Santa Catarina, 2016. Disponível em: <http://www.labeee.ufsc.br/sites/default/files/disciplinas/Apostila\%20Conforto \%20T\%C3\%A9rmico_2016.pdf>. Acesso em: 14 maio 2018.

LINDSLEY, M.; CADORETTE, M. Preventing heat-related illness in the workplace. Workplace Health \& Safety, Thousand Oaks, CA, USA, v. 63, n. 4, p. 192-192, 2015. DOI: 10.1177/2165079915588400. Disponível em: <https://journals.sagepub.com/doi/10.1177/2165079915588400>. Acesso em: 30 mar. 2019.

LYRA, Débora Santa Fé Monteiro. Aplicabilidade de índices de conforto térmico: um estudo de caso em Salvador - BA. 2007. 131 f. Dissertação (Mestrado em Engenharia Ambiental Urbana) - Escola Politécnica, Universidade Federal da Bahia, Salvador, 2007. Disponível em: <http://www.ppec.ufba.br/site/publicacoes/aplicabilidade-de-indices-deconforto-termico-um-estudo-de-caso-em-salvador-ba>. Acesso em: 14 maio 2018.

MICHAELIS, H. Dicionários Michaelis. São Paulo: Editora Melhoramentos Ltda., 2018. Disponível em: <http://michaelis.uol.com.br/>. Acesso em: 1 maio 2018. 
MILLER, V. S.; BATES, G. P. The thermal work limit is a simple reliable heat index for the protection of workers in thermally stressful environments. The Annals of Occupational Hygiene, Northamptonshire, UK, v. 51, n. 6, p. 553-561, 2007. DOI: 10.1093/annhyg/mem035. Disponível em:

<http://academic.oup.com/annweh/article/51/6/553/183107>. Acesso em: 30 mar. 2019.

MINISTÉRIO DO TRABALHO. Norma Regulamentadora 15 - Atividades Insalubres. 2014. Disponível em:

<http://trabalho.gov.br/images/Documentos/SST/NR/NR15/NR-15.pdf>. Acesso em: 10 maio 2018.

MINISTÉRIO DO TRABALHO. Norma Regulamentadora 17 - Ergonomia. 2007. Disponível em: <http://trabalho.gov.br/images/Documentos/SST/NR/NR17.pdf>. Acesso em: 10 maio 2018.

MINISTÉRIO DO TRABALHO. Norma Regulamentadora 7 - Programa de Controle Médico de Saúde Ocupacional. 2013. Disponível em:

<http://trabalho.gov.br/images/Documentos/SST/NR/NR7.pdf>. Acesso em: 10 maio 2018.

MINISTÉRIO DO TRABALHO. Norma Regulamentadora 9 - Programa de Prevenção de Riscos Ambientais. 2017. Disponível em:

<http://trabalho.gov.br/images/Documentos/SST/NR/NR-09.pdf>. Acesso em: 10 maio 2018.

MORRISON, S. F.; NAKAMURA, K. Central mechanisms for thermoregulation. Annual Review of Physiology, Palo Alto, CA, USA, v. 81, n. 1, p. 285-308, 2019. DOI: 10.1146/annurev-physiol-020518-114546. Disponível em: <http://www.annualreviews.org/doi/10.1146/annurev-physiol-020518-114546>. Acesso em: 18 abr. 2019.

MOURA, L. F.; XAVIER, A. A. P. Sensação térmica e ações para o conforto térmico: um estudo de caso. R. Gest. Industr., Ponta Grossa, v. 8, n. 2, p. 209-228, 2012. DOI: 10.3895/S1808-04482012000200009. Disponível em: <http://periodicos.utfpr.edu.br/revistagi/article/view/970>. Acesso em: 20 maio 2018. 
OLIVEIRA, Ronald Fred Alves de. Proposta de complementação do método de avaliação da exposição ocupacional ao calor: estudo de caso da atividade de manutenção de linhas energizadas. 2009. 132 f. Tese (Doutorado em Engenharia de Produção) - Programa de Pós Graduação em Engenharia de Produção, Universidade Federal da Paraíba, João Pessoa, 2009. Disponível em: <http://tede.biblioteca.ufpb.br:8080/handle/tede/5229>. Acesso em: 14 maio 2018.

OSHA. OSHA Sawmill eTool: Cold Stresses Descriptions of Hazards and Controls. Disponível em <http://www.osha.gov/SLTC/etools/sawmills/cold.html>. Acesso em: 10 abr. 2018.

SANTOS, F. L.; FIALHO, F. A. P.; CAVALCANTE, A. L. B. L. Análise ergonômica do trabalho de operadores de máquinas de esmaltagem - uma comparação entre tecnologias. R. Gest. Industr., Ponta Grossa, v. 7, n. 1, p. 107-122, 2011. DOI: 10.3895/S1808-04482011000100006. Disponível em: <http://periodicos.utfpr.edu.br/revistagi/article/view/408>. Acesso em: 20 maio 2018.

SANTOS, J. W.; MONTEIRO, L. F. Avaliação das condições de trabalho de agentes de bagagem e operadores de rampa de um aeroporto brasileiro. R. Gest. Industr., Ponta Grossa, v. 13, n. 1, p. 38-56, jan./mar. 2017. DOI: 10.3895/gi.v13n1.5021. Disponível em: <http://periodicos.utfpr.edu.br/revistagi/article/view/5021>. Acesso em: 20 maio 2018. crossref

TERRA, S. X.; FERREIRA, J. R. P.; CAMPOS, L. B.; COELHO, M. M.; FRANZ, L. A. S. Investigação quanto aos índices de conforto térmico em uma indústria de sorvetes. R. Gest. Industr., Ponta Grossa, v. 15, n. 1, p. 101-119, jan./mar. 2019. DOI: 10.3895/gi.v15n1.8394. Disponível em: <http://periodicos.utfpr.edu.br/revistagi/article/view/8394>. Acesso em: 18 abr. 2019.

VANGELOVA, K.; DEYANOV, C.; VELKOVA, D.; IVANOVA, M.; STANCHEV, V. Heat stress in two manufacturing units. Acta Medica Bulgarica, Warsaw, Poland, v. 35, n. 2, p. 40-46, 2018. Disponível em: <http://hdl.handle.net/10861/138>. Acesso em: 30 mar. 2019.

WANG, H.; HU, S. Analysis on body heat losses and its effect on thermal sensation of people under moderate activities. Building and Environment, Elsevier, v. 142, p. 180-187, 2018. DOI: 10.1016/j.buildenv.2018.06.019. Disponível em: <http://www.sciencedirect.com/science/article/pii/S0360132318303627>. Acesso em: 18 abr. 2019. crossref 
WIDMAIER, E. P.; RAFF, H.; STRANG, K. T. Fisiologia Humana: Os Mecanismos das Funções Corporais. 9. ed. Rio de Janeiro: Guanabara Koogan, 2006.

WRIGHT, H. E.; MCLELLAN, T. M.; STAPLETON, J. M.; HARDCASTLE, S. G.; KENNY, G. P. Cortisol and interleukin- 6 responses during intermittent exercise in two different hot environments with equivalent WBGT. Journal of Occupational and Environmental Hygiene, Taylor \& Francis, v. 9, n. 4, p. 269-279, 2012. DOI: 10.1080/15459624.2012.666467. Disponível em: <http://www.tandfonline.com/doi/abs/10.1080/15459624.2012.666467>. Acesso em: 18 abr. 2019.

ZHANG, N.; CAO, B.; ZHU, Y. Effects of pre-sleep thermal environment on human thermal state and sleep quality. Building and Environment, Elsevier, v. 148, p. 600-608, 2019. DOI: 10.1016/j.buildenv.2018.11.035. Disponível em: <http://www.sciencedirect.com/science/article/pii/S0360132318307303>. Acesso em: 18 abr. 2019. crossref 
Recebido: 14 Jun. 2018

Aprovado: 25 Abr. 2019

DOI: $10.3895 /$ gi.v15 n2.8418

Como citar:

ROSA, V. C.; LIMA, L. E. M. O estresse térmico visto como um risco ocupacional. R. Gest. Industr. Ponta Grossa, v. 15, n. 2, p. 53-73, Abr/Jun 2019. Disponível em: <http://periodicos.utfpr.edu.br/rgi>. Acesso em: $\mathrm{XXX}$.

Correspondência:

Victor Cupertino Rosa

Rua Padre Cesar de Buss, número 312, bairro Orfãs, Ponta Grossa, Paraná, Brasil.

Direito autoral: Este artigo está licenciado sob os termos da Licença Creative Commons-Atribuição 4.0 Internacional.

(c) (1) 